\title{
Zum Konzept des magischen Menschen bei Theodor-Wilhelm Danzel
}

\author{
Uwe Wolfradt
}

Eingegangen: 19. Mai 2020 / Angenommen: 29. Juni 2020 / Online publiziert: 13. August 2020

(C) Der/die Autor(en) 2020

Zusammenfassung Der Ethnologe Theodor Wilhelm Danzel (1886-1954) entwirft in den 1920er-Jahren ausgehend von der Völker- und Ganzheitspsychologie eine kulturpsychologische Strukturtheorie des Religiösen. In diesem theoretischen Ansatz folgt die psychische Struktur des Bewusstseins als Beziehung zwischen Subjekt und Objekt einer kulturellen Stufenentwicklung (von der primitiven zur Hochkultur). Hierbei nimmt Danzel mit stetiger Kulturentwicklung eine Abnahme der Komplexität und Subjektivität, eine Zunahme von Objektivität und Differenzierung an. Dem magischen Menschen (Homo divinans) stellt er den technischen Menschen (Homo faber) entgegen. In der Innenwelt des magischen Menschen ist das Objektiv-Dingliche von der Subjektivität durchdrungen (Subjektivierung des Objektiven), was sich dadurch bemerkbar macht, dass die Dinge wandelbar sind. Dinge besitzen einen inneren Bedeutungscharakter; sie sind beseelt. Der seelische Gehalt von Dingen ist ferner übertragbar, d. h. als Substanz kann er auf andere übertragen werden (wie z. B. die Kraft des Mana in Melanesien). Im Beitrag wird zunächst in die Strukturtheorie Danzels mit ihren geistigen Strömungen in der ersten Hälfte des 20. Jahrhunderts eingeführt. Anschließend wird die in seiner Theorie zentralen Figur des Homo divinans dargestellt und auf ihre Bedeutung für die heutige Religionsforschung überprüft. Hierbei soll gezeigt werden, dass die Dichotomie Homo divinans und Homo faber auch noch heute in der Psychologie unter anderer Bezeichnung an Bedeutung gewinnt.

Schlüsselwörter Ethnologie $\cdot$ Ganzheitspsychologie $\cdot$ Homo divinans · Kognition · Emotion

U. Wolfradt $(\triangle)$

Institut für Psychologie, Martin-Luther-Universität Halle-Wittenberg, Emil-Abderhalden-Str.

26-27, 06108 Halle (Saale), Deutschland

E-Mail: uwe.wolfradt@psych.uni-halle.de 


\title{
On the concept of magic man in Theodor-Wilhelm Danzel
}

\begin{abstract}
In the 1920s, the ethnologist Theodor-Wilhelm Danzel (1886-1954) developed a cultural-psychological theory of the structure of religion based on cultural psychology and holistic psychology. In this theoretical approach, the psychic structure of consciousness, as the relationship between subject and object, follows a cultural evolution (from primitive to high culture). In this process, Danzel assumes a decrease in complexity and subjectivity, and an increase in objectivity and differentiation, as culture is constantly developing. He contrasts the magic man (Homo divinans) with the technical man (Homo faber). In the inner world of the magic man, the object-material is permeated by subjectivity (subjectivization of the objective), which becomes apparent in the fact that things are changeable. Things possess an inner meaningful character; they are animated. The mental content of things is also transferable, i.e. as a substance it can be transferred to others (e.g. the power of mana in Melanesia). In this article, Danzel's structural theory, with its intellectual currents of the first half of the twentieth century, will be introduced. Subsequently, the central figure of his theory, Homo divinans, is presented and examined for its significance for contemporary religious research. The aim is to show that the dichotomy Homo divinans and Homo faber is still gaining importance in psychology today under a different name.
\end{abstract}

Keywords Ethnology $\cdot$ Holistic psychology $\cdot$ Homo divinans $\cdot$ Cognition $\cdot$ Emotion

Mit seinen Schriften gehört Theodor Wilhelm Danzel (1886-1954) zu jenen Vertretern, die der Struktur- und Ganzheitspsychologie Felix Kruegers (1874-1948) wie den Lehren von Alfred Vierkandt (1867-1953) und von Lucien Lévy-Bruhl (1857-1939) wichtige Anregungen entnahmen und auf dem Feld der Religionsethnologie und Mythologie fruchtbar anwenden konnten. Er war aber auch maßgeblich durch Wilhelm Wundt geprägt, hielt in seinen Werken stets an dem Begriff der Völkerpsychologie fest, obgleich er ihn entwicklungs- und strukturpsychologisch deutete. Danzel, als Vertreter der psychologischen Richtung in der Ethnologie, entwickelte seinerzeit mit der Gegenüberstellung von magischem Menschen (Homo divinans) und rational-technischem Menschen (Homo Faber) einen eigenständigen Ansatz in der Ethnologie. Hierbei kombiniert er naturmythologische Erklärungen der indigenen Völker (z. B. Veränderung kosmischer Systeme - Aufgehen von Mond und Sonne) mit völkerpsychologischen und psychoanalytischen Theorien (Projizierung des Subjektiven in das Gegenständliche) zu einem religionstheoretischen Ansatz der Wirklichkeitserkenntnis bei indigenen Völkern. Auch nach 100 Jahre mag das Konzept des magischen Menschen (Homo divinans) von Danzel in den heutigen Kulturwissenschaften befremdlich anmuten. Im Kontext von Psychologie, Ethnologie und Soziologie entstand eine Denkweise über Religion, die sich zum einen an den Zeitströmungen des Evolutionismus und zum anderen an der aufkommenden Struktur- und Ganzheitslehre orientierte. Insbesondere religiöse Phänomene wie Besessenheitskulte oder fremde Rituale beschäftigten die Kulturwissenschaftler seiner Zeit. Sie fügten sich leichter in die zeitgemäßen lebensphilosophischen Modelle, um 
das fremdartige Erleben und Verhalten indigener, seinerzeit als primitiv bezeichneter Völker besser zu verstehen. Danzels Dichotomie zwischen einem Typus der affektiven Hinwendung auf das Religiöse (Homo divinans) und einem Typus der rationalkognitiven Behandlung religiöser Phänomene (Homo faber) scheint im heutigen religionswissenschaftlichen Diskurs keine Rolle mehr zu spielen (vgl. Schmidt, 2008) oder eher zu historischen Betrachtungen anzuregen (vgl. Streck, 2013). Dabei fand diese Dichotomie durchaus Niederschlag in der zeitgenössischen Kulturphilosophie. Ernst Cassirer schreibt in seinem Beitrag „Form und Technik“ (1930, S. 28):

Der Mensch der Frühzeit und der der späteren Stufe scheiden sich, wie sich die Magie von der Technik unterscheidet: jener läßt sich als Homo divinans, dieser als Homo faber bezeichnen. Der gesamte Entwicklungsgang der Menschheit stellt sich alsdann als ein in zahllosen Zwischenformen sich vollziehender Verlauf dar, kraft dessen der Mensch von der Anfangsstufe des Homo divinans in die des Homo faber übergeht.

Cassirer ist einer der wenigen, die den Ansatz von Danzel aufgreifen und diesen für das Verhältnis zwischen Natur und Technik fruchtbar machen.

Im vorliegenden Beitrag soll das Religions- und Kulturverständnis Theodor-Wilhelm Danzels als psychologisch geschulten Vertreter des Strukturansatzes am Beispiel seines Konzepts des magischen Menschen nachgezeichnet werden. Schließlich soll an einen Kulturwissenschaftler erinnert werden, dessen Überlegungen auch heute noch in der Ethnologie und Kulturpsychologie eine Aktualität zukommt. So finden sich in der Persönlichkeitstheorie von Epstein (1990) zwei Verarbeitungsmodi, die der Unterteilung von Danzel zu entsprechen scheinen: ein rationaler und ein intuitiverlebnisbezogener Zugang zum Wirklichkeitsverständnis.

\section{Der Lebensweg von Theodor-Wilhelm Danzel}

Um den theoretischen Ansatz von Danzel besser zu verstehen, soll zunächst kurz der Lebensweg Danzels skizziert werden: Theodor-Wilhelm Danzel wird am 17. Februar 1886 in Hamburg geboren. Nach seiner Schulzeit studiert er ab 1906 Anthropologie und Völkerkunde zunächst an der Universität Göttingen, ab 1908 in Berlin und 1909 in Leipzig. Seine Lehrer sind die Ethnologen Paul Ehrenreich (1855-1914), Felix v. Luschan (1854-1924) sowie die Soziologen Georg Simmel (1858-1918), Ferdinand Tönnies (1855-1936) und Alfred Vierkandt (1867-1953) in Berlin; der Psychologe Wilhelm Wundt (1832-1920), der Ethnologe Karl Weule (1864-1924), der Kulturhistoriker Karl Lamprecht (1856-1915), der Geograph Joseph Partsch (1851-1925) sowie der Ägyptologe Georg Steindorff (1861-1951) in Leipzig. Diese sehr verschiedenen disziplinären Einflüsse münden im Jahr 1911 in seine Dissertationschrift „Die Anfänge der Schrift“, die von Weule und Lamprecht an der alma mater lipsiensis betreut wird. Danzel nimmt diese Erfahrungen aus der Psychologie, der Soziologie und den Kulturwissenschaften auf und beginnt eine wissenschaftliche Karriere in der Völkerkunde. Die Völkerkunde ist zu Beginn des 20. Jahrhundert noch ein vollkommen neues akademisches Fach. Er kehrt in seine Heimatstadt Hamburg zurück, wo er sich 1923 an der Universität Hamburg habilitiert; 1928 erhält 
er die Bezeichnung außerordentlicher Professor. Das Hamburger Museum für Völkerkunde bietet Danzel (1922) eine Stelle als freiwilliger Mitarbeiter, ab 1924 wird er schließlich ständiger Mitarbeiter und Kustos sowie Leiter der Allgemeinen und Afrika-Abteilung des Hamburger Museums für Völkerkunde. Im Auftrag des Museums unternimmt er Studienreisen nach Amerika (9. August bis 10. Dezember 1928), um dort am 23. Internationalen Amerikanisten-Kongress (17. September 1928) teilzunehmen und Museen aufzusuchen. Er begegnet den amerikanischen Kulturanthropologen Franz Boas (1858-1942) in New York, anschließend besucht er Mexiko. Von der chinesischen Regierung wird Danzel 1931 gebeten, sich am Aufbau einer Völkerkundeabteilung an der Universität Nanking zu beteiligen. Die Rückreise nutzt er für Aufenthalte in Japan, den Philippinen und in Ceylon. Aufgrund seiner jüdischen Herkunft (der Großmutter väterlicherseits) wird ihm zu Beginn der NSZeit zunächst die Lehrbefugnis an der Universität Hamburg entzogen, sodann wird er gezwungen, seine Museumstätigkeit aufzugeben (vgl. Fischer, 1990). Er wird jedoch wieder eingestellt, gewinnt aber nicht seine frühere Schaffenskraft zurück. Er verstirbt am 16. November 1954 nach langer Krankheit in Hamburg (vgl. Vorwort Westermann, Danzel, 1967; Nachlass Danzel, NTWD).

\section{Grundlagen des ethnopsychologischen Denkens bei Theodor-Wilhelm Danzel}

Danzel (1921, 1928, 1930) bezieht sich in seinem Denken im Wesentlichen auf Vertreter der Leipziger Völkerpsychologie wie Wilhelm Wundt und den Ganzheitspsychologen Felix Krueger, ebenso auf den Berliner Kultursoziologen Alfred Vierkandt sowie den französischen Philosophiehistoriker Lucien Lévy-Bruhl (Danzel, 1928, S. 9). In der Völkerpsychologie Wilhelm Wundts sieht Danzel einen wichtigen Wegbereiter, kulturelle Aspekte der Psychologie in den Vordergrund zu heben. So vertrat Wundt eine evolutionäre Kulturtheorie, die sich durch vier Stadien unterschied: (1) eine Primitivkultur, die durch einen Zauber- und Dämonenglauben, reduzierte Sprache und gegenständliches Denken sowie frühe Formen der Kunstschaffung geprägt ist; (2) dem Totemismus, der durch Totem- und Tabuvorstellungen charakterisiert ist und sich durch Ahnenkulte und Initiationsriten auszeichnet; (3) der Heroenzeit, die durch kosmogonische und theogonische Mythen (mit Helden und Göttern), Rechtskodifizierungen, Ansätze zur Gesellschaftswerdung mit sozialer Stratifizierung und Städtegründungen charakterisiert ist, und (4) der Humanität, in der Weltreiche, Weltkulturen, Weltreligionen und Weltgeschichte vorherrschen (1913). Ideen von Wundt finden sich immer wieder im Werk von Danzel insbesondere zur Mythologie und zum mythischen Bewusstsein. Wundt sieht zwei Faktoren, die für die Entwicklung von Mythus und Religion zentral sind. Zum einen nimmt er eine Differenzierung der Seelenvorstellungen an, die erst eine höhere Seelenmythologie ermöglichen, und zum anderen tragen kultische Handlungen (Kultus) zur sozialen Normierung des religiösen Denkens bei. Wundt findet schließlich zur Unterscheidung zwischen Körperseele (gebundene Seele), die an die leibliche Konstitution gebunden ist, und Psyche (freie Seele), die über Jenseitsphantasien mit der Erfahrungswelt verbunden ist. Nur so waren für Wundt religiöse Phänomene wie See- 
lenwanderung, Besessenheit und ekstatische Ergriffenheit nachvollziehbar. Wundt übertrug seine Entwicklungstheorie auf die Religion, indem er verschiedene Stadien der menschlichen Phantasietätigkeit als „Entwicklungsstufen des Seelenglaubens“ postulierte. Religion ist ihm zufolge wie der Mythus als Produkt der menschlichen Phantasie aufzufassen (Wundt, 1905, S. 4, 1906, S. 1). Religion differenziere sich über verschiedene Entwicklungsstufen zu höheren, monotheistischen Religionsformen (vgl. Allesch 2006). Danzel hält an dem Begriff der Völkerpsychologie von Wundt fest und orientiert sich an dessen Stufenmodell, indem er einen Wandel von einer vorwiegend magischen Stufe zu einer vorwiegend technischen Stufe annimmt, allerdings ohne hier eine Wertung vornehmen zu wollen (1930, S. 12).

Danzel verfolgt in den 1920er-Jahren in den Kulturwissenschaften einen Streit darüber, ob indigene Völker zu logisch-rationalem Denken fähig sind. Lévy-Bruhl (1921) vertritt seinerzeit die Auffassung, dass das Denken der Naturvölker prälogisch, nicht unlogisch sei. Mittels einer philosophischen Textanalyse versucht er, die Aussagen von Naturvölkern aus westlich-rationalistischer Perspektive zu interpretieren, d.h. er führt selbst keine eigenen Feldstudien durch, sondern verlässt sich auf das Material von anderen europäischen Reisenden oder Missionaren. Eine Trennung zwischen Konkret-Gegenständlichem und Abstrakt-Begrifflichem scheint ihm zufolge bei den indigenen und seinerzeit primitiv genannten Völkern nicht vorzuliegen. Durch participation mystique projiziert der Indigene seine Emotionen auf Objekte. „Die Naturvölker werden hier durch einen besonderen Gefühlssinn geleitet. Die Erfahrung entwickelt und verfeinert ihn, und er kann unfehlbar werden, ohne irgendetwas mit Denkoperationen im eigentlichen Sinne zu tun zu haben“, so Lévy-Bruhl (1927, S. 350f.). Er distanziert sich jedoch später von vielen seiner früher getroffenen Aussagen zum prälogischen Gehalt des primitiven Denkens (vgl. Evans-Pritchard, 1981). Der britische Sozialanthropologe Edward E. Evans-Pritchard (1902-1973) geht dieser Frage ebenfalls nach und zeigt sehr eindrucksvoll am Beispiel der afrikanischen Azande, dass diese logisch denken können, aber trotzdem eine magische Interpretation wählen (vgl. Heinz, 1997). Als berühmtes Beispiel für die Spannung zwischen magischem und logischem Denken gilt folgender Fall: Ein Mann sucht unter einem Getreidespeicher Schatten vor der Sonne, als plötzlich der Speicher über den Mann zusammenstürzt. Die Azande glauben in diesem Fall an einen Hexenzauber. Sie verstehen, dass der Speicher durch Termiten zum Einsturz kam (logische Kausalität). Allerdings fragen sie sich, warum der Mann gerade in diesem Moment sich dort hinsetzt, wo es zum Einsturz kommt. Letzteres erklären sie sich magisch (Evans-Pritchard, 1976, S. 22f.).

Vierkandt (1937) versucht Lévy-Bruhls Gruppenanschauungen (représentations collectives) entwicklungspsychologisch $\mathrm{zu}$ deuten, indem er diese als vorrational und komplex, als von Gefühls- und Triebelementen vollkommen bestimmt betrachtet. Lévy-Bruhl wendet sich entsprechend der französischen Durkheim-Schule einer objektiven Beschreibung der Inhalte von Gruppenanschauungen und deren sozialer Funktion zu und verlässt somit die Grundlage der reinen psychologischen Analyse. Vierkandt kritisiert diese stark rationalistische Perspektive auf das Denken der Naturvölker bei Lévy-Bruhl. Am Beispiel der Zauberhandlungen stellt Vierkandt seine Auffassungen den Ansichten von Lévy-Bruhl entgegen. Demzufolge sind magische Rituale als rein triebhafter Ausdruck bestimmter Gemütsbewegungen und Gesin- 
nungen zu verstehen. Ihnen kommt in ihrem ursprünglichen Sinn kein eigentlicher Handlungszweck zu. Zauberhandlungen sind Vierkandt zufolge vielmehr Manifestationen bestimmter Affekte und Stimmungen. Er interpretiert die Zauberei als ein sozialpsychologisches Phänomen, da der Mensch als Zoon politikon stets auf die soziale Umgebung einzuwirken versucht. Die Zauberei ließe sich somit als soziale Ausdrucks- und Zweckhandlung charakterisieren, die ein soziales Bedürfnis zum Ausdruck bringt, das aber auf biologischen Trieben basiert (vgl. auch Wolfradt, 2011). Danzel macht sich diese Auffassung von Vierkandt zu eigen, nachdem das Denken des indigenen Menschen durch seine soziale Gemeinschaft geprägt ist, wobei die biologischen Erklärungen für das Denken und Verhalten von ihm nicht überbewertet werden (1924, S. 5).

Während die frühen Arbeiten von Danzel noch eng an die Wundtsche Völkerpsychologie, aber auch an Vierkandts Kultursoziologie angelehnt sind, gewinnt für Danzel zu Beginn der 1920er-Jahren ebenfalls die Struktur- und Ganzheitspsychologie Felix Kruegers an Bedeutung. Der Strukturbegriff Kruegers geht auf Wilhelm Dilthey (1833-1911) zurück, an dessen Vorlesungen Danzel als Student an der Berliner Universität teilnimmt. Dilthey entwendet den Begriff der Struktur den Naturwissenschaften und verstand hierunter ein ganzheitliches Gefüge, das dem Muster biologischer Organismen gleicht. Struktur bezog er auf die Biographie (persönliche Lebensgeschichte). Leben und Erleben lassen sich ihm zufolge drei Bedingungen zuordnen: (1) Zeitlichkeit: Kontinuitätsbezug des Erlebens, (2) Geschichtlichkeit: kultureller und anthropologischer Erlebensbezug und (3) Unmittelbarkeit: (emotionaler, selbstbezogener und ursprünglicher Erlebensbezug; vgl. Pongratz 1984, S. 248-252). Dilthey versteht die psychische Struktur als einen inneren Zusammenhang von Teilen seelischer Vorgänge, indem er feststellt (1924, S. 66):

Sie [die psychische Struktur] ist die Anordnung, nach welcher psychische Tatsachen von verschiedener Beschaffenheit im entwickelten Seelenleben durch eine innere erlebbare Beziehung miteinander verbunden sind. Die Grundform dieses seelischen Zusammenhangs ist dadurch bestimmt, dass sich alles psychische Leben von seinem Milieu bedingt findet und rückwärts auf dies Milieu zweckmäßig einwirkt.

In Orientierung an der strukturalen Entwicklungspsychologie von Krueger betrachtet Danzel (1930) Kultur als einen ganzheitlichen biologischen wie sozialpsychischen Organismus, der sich fortwährend weiterentwickelt. Gebilde wie Kunstwerke, Sprache, Schrift, technische, soziale und wirtschaftliche Institutionen, die von Menschen gestaltet werden, sind als heterogene Elemente in der Kulturgemeinschaft miteinander verbunden und aufeinander bezogen. Ihnen kommt eine Funktion im Sinn des Ganzen zu. Der Mensch kann in diesem Kontext als aktiver Gestalter seiner Umwelt, seiner Kultur betrachtet werden. Danzel widersetzt sich einer mechanistischen Erklärungsweise, ,die das Ganze als eine Summe von einzelnen eindeutigen und konstanten Elementen, Momenten genügend verständlich gemacht zu haben glaubt“" (1921, S. 51f.). Kulturgemeinschaften sind vielmehr dynamisch und organisch zu verstehen, die in einem zeitlichen Nacheinander und einem räumlichen Nebeneinander charakteristische Formen und Gestalten ausbilden. 
Dem mythisch-religiösen Gebiet kommt ihm zufolge eine wichtige Bedeutung für die kulturelle Betätigung zu. Sowohl Religion als auch Magie besitzen die Funktion, Gottheiten oder Dinge zu weihen und zu heiligen. Hinter jeder religiösen Übung steht der Wunsch, die Seinsgrenze zu überschreiten, d. h. in Kontakt mit einer göttlichen oder spirituellen Sphäre zu treten. Dahinter steht als ultimater Grund das Beschwichtigen von Ahnen und Geistern mittels Zeremonien, Kulten, Opfern und zauberischen Handlungen. Zudem werden Wirklichkeitsdeutung und Weltinterpretation durch religiöse Handlungen ermöglicht, die die Harmonie und Gewissheit zwischen dies- und jenseitiger Welt aufrechterhalten.

Ein weiterer prägender Einfluss auf Danzel geht von Herbert Spencer (1820-1903) aus, der den Evolutionismus in die Psychologie und die Soziologie brachte und einen nachhaltigen Einfluss auch auf Wundt und Krueger ausübte. Spencer $(1886,1887)$ betrachtet das menschliche Bewusstsein als das Resultat eines sozialen Anpassungsprozesses, in dem alle geistigen Tätigkeiten einer Differenzierung und Integrierung von Bewusstseinszuständen unterliegen. Das Erkennen von Unterschieden in den Zuständen und der Verbindung verschiedener Zustände (unterschiedlicher Komplexität) kann als ein Prozess der Adaptation der Eindrücke der Außenwelt an die Innenwelt verstanden werden. Die Unterscheidung primitiv (einfach gegliedert) versus komplex (vielseitig gegliedert) findet auf Kulturstufen Anwendung, indem undifferenzierte Gebilde zu differenzierten Strukturen werden (1887, S. 6). Seinerzeit ging man von der Perspektive aus, dass das Studium der Urvölker durch die Analyse des Denkens und Verhaltens der indigenen Völker der Gegenwart möglich wäre, ohne zu bedenken, dass die indigenen Völker natürlich auch schon einer kulturellen Entwicklung gefolgt sind (vgl. auch Wolfradt 2013).

\section{Zum Konzept des magischen Menschen (Homo divinans)}

Zentral für die wissenschaftliche Tätigkeit von Danzel ist die Psychologie des indigenen (,,primitiven“) Menschen, bei der er sich um eine objektive Beschreibung und eine historische Interpretation bemüht. Wichtige Vorarbeit für die Konzeption des Homo divinans leistet Danzel in seiner Dissertation „Die Anfänge der Schrift“ von 1912. Hier nimmt er eine völkerpsychologische Analyse von Schriftsystemen und Symbolen vor. Er stellt fest, dass die Bilderschrift ihren Ursprung in der Gebärde und der darstellenden Kunst hat und zunächst die Funktion hatte, magisch-religiöse Zeichen zu vermitteln. Geprägt durch den Evolutionismus in den Werken von Wundt sieht er die psychologischen Wurzeln für Schrift und Zeichen vornehmlich in Ausdrucksbewegungen, also triebhaften Äußerungen von inneren psychischen Zuständen, die unbewusst als Entladungen von Gefühlsspannungen im GraphischZeichnerischen Ausdruck finden. Er gelangt zur Überzeugung, dass indigene Menschen über sinnliche Vorstellungen verfügen, die komplexer, kollektiver und ungegliedert seien. Ihnen gelingt folglich keine Trennung zwischen Objekt und Subjekt, da der Gegenstand nicht gedacht, sondern erlebt wird (1912, S. 8):

Denn es werden, indem der Primitive das Objekt geistig erfasst und von diesem gleichsam erfasst wird, also eine emotionale Verschmelzung von großer In- 
nigkeit auftritt, in diesen umfassenden Vorstellungskomplex auch unvermeidlich andere subjektive Gefühle einfließen. Äußerliche, zufällige Beziehungen und Zusammenhänge der Dinge, durch den Augenblick gegebene Bewertungen werden durch den Geist noch nicht als solche als nur im Subjekt bestehend erkannt.

Er findet zur Unterscheidung von zwei idealtypischen Vertretern: den Homo divinans, einem magisch handelnden Menschen, der durch Komplexheit, Subjektivität und Emotionalität bestimmt ist, und den Homo faber, einem technisch handelnden Menschen, der sich intellektuell und bewusst differenzierend der Welt zuwendet (vgl. auch Danzel 1924, 1928).

Besonders die magischen Symbole dienen dem magischen Menschen aufgrund mangelnder Abstraktionsfähigkeit als Projektionsfläche für das eigene religiöse Erleben. Der Ursprung der magischen Symbole liegt in dem ideographischen Zeichnen, aus dem schließlich die Bilderschrift hervorging, die zunächst religiöse Motive zum Inhalt hatte. Danzel geht es nur um eine Charakterisierung der Erlebensperspektive von Menschen, ohne eine Bewertung vornehmen zu wollen. Hierzu hält er fest (1928, S. 19):

Wir dürfen die sog. Frühstufen, die sog. Primitiven und primitiveren Völker nicht als etwas Minderwertiges ansehen. Wir dürfen nicht in selbstgefälliger Anmaßung als gesichert annehmen, daß wir heutigen europäischen Menschen auf einem unbestrittenen Höhepunkte der Kultur ständen und nur mit einer gewissen Verachtung auf die sog. ,barbarischen' zurückgebliebenen, rohen Völkerschaften herabblicken könnten.

Danzel, der selbst keine ethnologische Feldforschung durchführte und sich zumeist auf Berichte von Völkerkundlern und Missionaren stützte, stellt aufgrund der Beobachtungen der nordamerikanischen Indianer der Bulu und der Sioux bei Völkerschauen im Hagenbeckschen Tierpark Hamburg Strukturverschiedenheiten zwischen Homo divinans und Homo faber fest. Hierzu schreibt er (1921, S. 60):

Die Struktur stellt sich als die Beziehung des Objektiven zum Subjektiven im Bewußtsein dar und ihre Wandlungen erweisen sich als im Laufe der Entwicklung zunehmende bewußtheitliche Differenzierung, zunehmende Intellektualität, zunehmende Objektivität, abnehmende Komplexheit des Bewußtseins, abnehmende Emotionalität, abnehmende Subjektivität.

Unterschiede für das Verhalten von Homo divinans und Homo faber führt Danzel auf die allgemeinen Strukturverschiedenheiten des Bewusstseins zurück. Krüger und auch Spencer folgend postuliert er Entwicklungsstufen, die sich durch ihren Grad der seelischen Differenziertheit versus Komplexität, Objektivität versus Subjektivität und Intellektualität versus Emotionalität auszeichnen. Am Anfang der menschlichen Entwicklung steht ihm zufolge ein Zustand der undifferenzierten Bewußtheitlichkeit, der primitiven Erlebensform, bei der die Dinge noch nicht durch kritische Distanzierung objektiviert sind. Dieses Bewusstsein entwickelt sich von der primitiven Kulturstufe zur hochkulturellen Stufe, indem es zunehmend objektiver und rationalintellektueller wird. Diese undifferenzierte Struktur manifestiert sich bei indigenen 
Völkern in Formen des Polytheismus, der Vorstellung mehrerer Beseeltheiten in der Umwelt (z. B. jedem Baum wird die Seele eines Ahnen zugeschrieben). Er geht soweit, das Psychische bei indigenen Völkern ganz infrage zu stellen, und schreibt in einer Abhandlung mit dem Titel „Die psychologische Bedeutung magischer Bräuche" (1922, S. 64):

Man kann zweifeln, ob die psychischen Prozesse des Primitiven, um die es sich hier handelt, die Bezeichnung ,psychisch“ überhaupt verdienen. Die Akte sind so komplex, dass es unmöglich ist, psychische und motorische zu trennen. Bewegungen sind integrierende Bestandteile eines psychische und physische Vollzüge umfassenden Gesamtaktes.

Und Danzel schreibt in einem weiteren Beitrag mit dem Titel „Die psychologischen Grundlagen der Mythologie“" zum mythischen Bewusstsein (1927, S. 496):

Der Mythos ist die besondere Anschauungsform und Erlebnisform des primitiven und primitiveren Menschen. Der primitive Mensch sieht, erlebt mythisch. Die Mythen sind ursprünglich keine Einkleidungen, Gleichnisse, Allegorien, bewußte Übertragungen menschlicher Geschehnissen insbesondere auf kosmische, so sehr sie uns als Außenstehenden so erscheinen mögen.

Der magische Mensch, Homo divinans, projiziert Danzel zufolge in das Ding bzw. Objekt das Subjektive, die Welt wird emotional beseelt oder spiritualisiert Dinge erhalten eine Sakralität. Durch die Subjektivierung des Objektiven wird die Kluft zwischen Gegenständlichem und Zuständlichem, zwischen Dinglichem und Seelischem überbrückt. Das beseelte Objekt kann für den Homo divinans etwas Außenweltliches erhalten, z. B. als Dämon mit negativen Kräften. Hierbei kann das Seelische als Kraft auf Materielles übertragen werden (wie ein Stoff, eine Substanz, Mana in Polynesien, Anthropophagie - das Essen des Herzens eines mutigen Kriegers).

Diese Komplexheit des indigenen Bewusstseins in Bezug auf den Mythos führt nach Danzel (1922) zum einen zu einer Vieldeutigkeit der mythischen Gestalten (z. B. lunare und gleichzeitig solare Züge bei der altmexikanischen Mondgöttin Tlacolteotl) und zum anderen zu einer Verteilung der Objekte oder Züge auf verschiedene mythische Gestalten.

Mythen sind psychologische Quellen für das magische Denken, um psychische Spannungen, Konflikte, Vorgänge und Zustände identifizieren zu können. Diese Konflikte werden in das Naturmystische introjiziert und symbolhaft ausgedrückt.

Für die Unterschiede zwischen verschiedenen Bewusstseinsstufen postuliert er keine rassisch-biologischen Gründe, wie dies seinerzeit üblich war, sondern betont die Bedeutung und Sinnhaftigkeit der jeweiligen Bewusstseinsstufe aus sich selbst heraus. Er spricht hier von einem unterschiedlichen Wirklichkeitsverständnis des magischen Menschen (Homo divinans). Und er hält fest (1930, S. 39):

Unsere Wirklichkeitserlebnisse entsprechen allerlei begrifflichen Unterscheidungsformen wie subjektiv und objektiv, Gegenstand und Zustand, Geist und Stoff usw. Diese begrifflichen Unterscheidungen verlieren für die Welt, wie sie der indigene Mensch erlebt, bis zu einem gewissen Grade - das zeigen eben sei- 
ne Mythen und Kultur, sein Dämonismus und seine magische Praxis - ihre Gültigkeit und Anwendbarkeit. Dagegen finden wir aber andere Unterscheidungen, die der Erlebniswelt des Primitiven gemäß sind, in Zusammenhang mit denen sich die magischen Handlungen als durchaus sinnvolle Vollzüge erweisen.

Danzel nennt in diesem Kontext als wesentliche Merkmale für das Bewusstsein des magischen Menschen (Homo divinans): Zum einen eine hohe Empfänglichkeit gegenüber Suggestionen und starke Einbildungskraft (z. B. Krankheit kann aus dem Körper herausgesaugt werden). Zum anderen wird die Bedeutung von Visionen und Träumen herausgestellt, so z. B. können Träume Dinge offenbaren, denen die Seele begegnet sei. Zusammenfassend kann für das kulturpsychologische Denkens Danzels festgestellt werden: (1) der Mensch kann sowohl als magisch (Homo divinans) als auch als rational-handelnd (Homo faber) aufgefasst werden; (2) dem mythischen Bewusstseins kommt eine große Bedeutung zu, d.h. der Mythos kann als eine menschliche Ausdrucksform, als eine Basis für die Imaginationskraft und als Grundlage für die zwischenmenschliche Kommunikation verstanden werden (z. B. Schöpfungsmythen, Märchen); (3) Denken und Handeln sind untrennbar miteinander verbunden, d.h. es wird eine Harmonie zwischen psychischen und leiblichkörperlichen Lebensprozessen postuliert und (4) Symbole, die dem Menschen seine spirituelle Existenz vermitteln, schaffen bewusst oder unbewusst einen weltlichen Bezug (z. B. religiöse Symbole).

\section{Conclusio}

Zeitgleich wie Danzel entwickelte Heinz Werner (1890-1964) in seiner Entwicklungspsychologie (1933) - ebenso an Felix Krueger orientiert - ähnliche Überlegungen, indem er der komplexen Struktur, die sich auf die Bedeutung einer Gegebenheit bezieht, die diffuse Struktur entgegensetzt, die sich dadurch auszeichnet, dass sie nur der Form nach eine Ungegliedertheit aufweist. Nach Werner ist der Naturmensch - ähnlich wie bei Danzel der Homo divinans - durch eine diffuse Wahrnehmung bestimmt, einer Unfähigkeit, aus singulären Informationen ein ganzheitlich gegliedertes Gesamtgefüge zusammenzusetzen. Werner hebt weiterhin hervor, dass diese mangelnde kognitive Integrationsfähigkeit zu einem starren Weltbild bei Naturvölkern führen muss. Ihm zufolge sind Phantasie und Erinnerungsvorstellungen von Naturvölkern in ihrer Bedeutung komplexer und ihrer Form nach diffuser als die der Kulturvölker. Folglich verfügt der Naturmensch nur über eine geringe Distanz zwischen der Welt innerer Zustände und äußerer Gegenstände, sodass beispielsweise die Unterscheidung zwischen magischer Traumwelt und Wirklichkeit nicht gelingt. Diese Undifferenziertheit der seelischen Funktionen beim indigenen Menschen lassen sich beim Fühlen und Wahrnehmen, Vorstellen und im Sinngehalt aller Erscheinungen nachweisen. Magische Handlungen und Gegenstände der sogenannten Primitiven sind diffus und komplex. Die hieraus resultierende Inkonstanz der Dinge führt zu einer Instabilität der Eigenschaften dieser Gegenstände und einer Übertragbarkeit der Eigenschaften auf andere Gegenstände und Personen. Werner sieht eine Ähnlichkeit dieser Denkweisen des indigenen Menschen auch bei Kin- 
dern und psychisch Kranken (z.B. Schizophrenen; Werner, 1933, S. 94f.). Auch Werner (1933) betrachtet die Wirkung der Gefühle auf die magischen Sphären bei den Naturmenschen (S. 321):

Die Dinge und Personen der Umwelt werden in der immer bewußter werdenden magischen Sphäre als dämonische Wirksamkeiten gefaßt, deren letzte Bedingung in dem Anteil liegt, welchen die Affekte an der Gestaltung der Umwelt haben. Die Welt wird zu magischen Wesenheiten, die das Widerspiel der Ängste und Wünsche des Menschen sind.

Die mangelnde Unterscheidung zwischen Subjektivität und Objektivität bei Kleinkindern fand in der genetischen Epistemologie des Schweizer Psychologen Jean Piaget (1896-1980) als ,,kindlichen Realismus“ Eingang, also der Vorstellung, dass der Begriff oder die Regel mit der bezeichneten Sache identisch sei. Der kanadische Kulturanthropologe Christopher Robert Hallpike (1979) greift diese Gedanken Piagets in seinem Buch Die Grundlagen primitiven Denkens (Foundations of Primitive Thoughts, 1979) auf und spricht von einem ,begrifflichen Realismus“ bei indigenen Völkern: So soll es kaum operative Korrekturen der Wahrnehmung bei indigenen Völkern geben, d.h. das, was wahrgenommen wurde, gilt auch als real. Ferner gibt es kaum Formen des deduktiven Folgerns, d. h. logische Beweisführung findet nicht statt. Weiterhin werden individuelle Erfahrungen nur selten zum Ausdruck gebracht und innere Zustände (wie Beweggründe, Gefühle) werden vom äußeren Verhalten her beurteilt. Zudem sind kollektive Vorstellungen in sozialen Institutionen eingebunden, die eine Unterscheidung zwischen Anschauungen und Tatsachen erschweren. Schließlich ist es generell schwer, im symbolischen Denken die subjektiven und objektiven Aspekte zu trennen (z. B. im Gegensatz zur Sprache).

Es muss kritisch angemerkt werden, dass die eurozentrische Sichtweise einer klaren Dichotomisierung zwischen magisch-primitivem und rational-logischem Denken heute aus kulturwissenschaftlicher Perspektive nicht mehr aufrechterhalten werden kann. Vielmehr handelt es sich um komplementäre Sichtweisen, die je nach kulturellem Kontext vorherrschend sein können. So verweist etwa Descorla (2011) in seinen Überlegungen darauf hin, dass die strenge westliche Unterscheidung zwischen Kultur und Natur in einer globalisierten Welt nicht mehr haltbar ist, da viele Völker einen fließenden Übergang zwischen Natur und Kultur annehmen (z.B. Beseeltheit der Natur, verwandtschaftliche Beziehungen zwischen Menschen und Tieren). Indigene Menschen sind genauso $\mathrm{zu}$ rationalem wie magischem Denken in $\mathrm{Ab}$ hängigkeit von den Erfordernissen des jeweiligen Handlungskontexts fähig wie der Mensch der Zivilisation zum magischen Denken (z.B. paranormale Überzeugungen). Schon Edward Burnett Tylor (1832-1917) etablierte 1871 mit seiner SurvivalTheorie die Annahme, dass magisches Denken der Vergangenheit in die Kultur der Gegenwart reicht und als Form magischen Denkens und Sitten zutage tritt (z. B. alte vorchristliche Gebräuche und Mythen; vgl. auch Ratnapalan 2008).

Obgleich die evolutionistischen Annahmen von Danzel dem Zeitgeist entsprachen, war er bemüht, den Besonderheiten des psychischen Erlebens indigener Völker gerecht $\mathrm{zu}$ werden und verwies recht früh auf die Gefahren für das Bestehen von bedrohten ethnischen Minderheiten auf der Welt durch die westliche $\mathrm{Zi}$ vilisation. Die Typologie vom Homo divinans und Homo faber kann dann einen 
fruchtbaren Ansatz von komplementären Denkungsstilen für die heutige Religionsforschung darstellen, wenn psychologische Phänomene sowohl aus einer kognitivheuristischen wie aus einer emotional-intuitiven Perspektive betrachtet werden, wie dies Untersuchungen von Epstein et al. (1990, 1996) nahelegen. Die Cognitive-Experiential Self-Theory (CEST; Epstein 1990) postuliert zwei unabhängig, aber auch interaktiv operierende Informationsverarbeitungssysteme: ein analytisch-rationales sowie ein intuitiv-experientielles. Das intuitiv-experientielle System beinhaltet eine automatisierte, holistische, und affekt-assoziationistische Informationsverarbeitung. Das analytisch-rationale System steht demgegenüber für eine bewusste und logischschlussfolgernde Informationsverarbeitung. Evolutionär ist das intuitiv-experientielle System vor dem analytisch-rationalen System entstanden. In diesem Kontext findet man die Dichotomie von Danzel wieder: So kann der Homo divinans dem intuitiv-experientiellen Verarbeitungsmodus und der Homo faber dem analytischrationalen Verarbeitungsmodus zugeordnet werden. Verschiedene Studien zeigten bezogen auf religiöse Phänomene, dass das intuitiv-experientielle System stärker mit Religiosität (Razmyar und Reeve 2013) und mit paranormalen Überzeugungen und Erfahrungen (Wolfradt et al. 1999) zusammenhängt. Diese neuen theoretischen Überlegungen und empirischen Befunde in der Psychologie zeigen, dass die Grundgedanken von Theodor-Wilhelm Danzel auch heute in neuem Gewand noch aktuell sind. Der Homo divinans wie der Homo faber stehen für verschiedene aufeinander bezogene Weltauffassungen und Verständnisweisen von Wirklichkeit, die jeweils ihre notwendige Funktion für den Umgang mit unkontrollierbaren Widrigkeiten des Lebens haben (Naturkatastrophen, plötzliche Krisen wie Krankheit und Tod). Das Unkontrollierbare der Welt ist das Geheimnisvolle in der erlebten Wirklichkeit, wo durch Wissen keine Gewissheit erbracht werden kann, sondern nur das MagischIntuitive des religiösen Glaubens Ruhe und Klarheit bringt. Boesch (1983) betont die Bedeutung der Magie für die Herstellung von Ordnung und Struktur in unserer Welt (S. 97):

Magie baut auf die Grundüberzeugung, daß unsere Welt eine - in irgendeiner Weise - geordnete sei, und daß unser Wohl und Wehe, im einzelnen wie im gesamten, davon abhänge, wie sehr wir unser Handeln diesen Ordnungen einfügen.

Funding Open Access funding provided by Projekt DEAL.

Open Access Dieser Artikel wird unter der Creative Commons Namensnennung 4.0 International Lizenz veröffentlicht, welche die Nutzung, Vervielfältigung, Bearbeitung, Verbreitung und Wiedergabe in jeglichem Medium und Format erlaubt, sofern Sie den/die ursprünglichen Autor(en) und die Quelle ordnungsgemäß nennen, einen Link zur Creative Commons Lizenz beifügen und angeben, ob Änderungen vorgenommen wurden.

Die in diesem Artikel enthaltenen Bilder und sonstiges Drittmaterial unterliegen ebenfalls der genannten Creative Commons Lizenz, sofern sich aus der Abbildungslegende nichts anderes ergibt. Sofern das betreffende Material nicht unter der genannten Creative Commons Lizenz steht und die betreffende Handlung nicht nach gesetzlichen Vorschriften erlaubt ist, ist für die oben aufgeführten Weiterverwendungen des Materials die Einwilligung des jeweiligen Rechteinhabers einzuholen.

Weitere Details zur Lizenz entnehmen Sie bitte der Lizenzinformation auf http://creativecommons.org/ licenses/by/4.0/deed.de. 


\section{Literatur}

Allesch, Christian G. 2006. Das Forschungsfeld ,Mythus“ bei Wilhelm Wundt. In Wilhelm Wundts anderes Erbe, Hrsg. G. Jüttemann, 156-166. Göttingen: Vandenhoeck \& Ruprecht.

Boesch, E.E. 1983. Das Magische und das Schöne. Zur Symbolik von Objekten und Handlungen. StuttgartBad Cannstatt: frommann-holzboog.

Cassirer, E. 1930. Form und Technik. In Kunst und Technik, Hrsg. L. Kestenberg, 1561. Berlin: Wegweiser. Danzel, T.-W. 1912. Die Anfänge der Schrift. Leipzig: Voigtländer.

Danzel, T.-W. 1921. Prinzipien und Methoden der Entwicklungspsychologie. Grundlinien einer psychologischen Entwicklungsgeschichte von Kultur und Gesellschaft. In Handbuch der biologischen Arbeitsmethoden Abt. VI, Teil C., Hrsg. E. Abderhalden, 45-108. Berlin: Urban \& Schwarzenberg.

Danzel, T.-W. 1922. Die psychologische Bedeutung magischer Bräuche. Psychologische Forschung 2:61-64.

Danzel, T.-W. 1924. Kultur und Religion des primitiven Menschen. Einführung in die Hauptprobleme der allgemeinen Völkerkunde und Völkerpsychologie. Stuttgart: Strecker \& Schröder.

Danzel, T.-W. 1927. Die psychologischen Grundlagen der Mythologie. In Festschrift Meinhof. Glückstadt/ Hamburg: Augustin.

Danzel, T.-W. 1928. Der Magische Mensch (Homo Divinans). Vom Wesen der primitiven Kultur. Potsdam: Müller \& Kiepenheuer.

Danzel, T.-W. 1930. Gefüge und Fundamente der Kultur. Vom Standpunkte der Ethnologie (Prolegomena). Hamburg: Friederichsen, De Gruyter.

Danzel, T.-W. 1967. Ethnologische Kulturkunde. Versuch einer universalen Systematik der Kulturwissenschaften. Hamburg: Christians. Hrsg. v. H. Danzel \& A. Westermann.

Descorla, P. 2011. Jenseits von Natur und Kultur. Berlin: Suhrkamp.

Dilthey, W. 1924. Ideen über eine beschreibende und zergliedernde Psychologie (1894). In Wilhelms Diltheys Gesammelte Schriften, Bd. V, 139-240. Leipzig \& Berlin: Teubner.

Epstein, S. 1990. Cognitive experiential self theory. In Handbook of personality: theory and research, Hrsg. L. Pervin, 165-192. New York: Guilford.

Epstein, S., R. Pacini, V. Denes-Raj, und H. Heier. 1996. Individual differences in intuitive-experiential and analytical-rational thinking styles. Journal of Personality and Social Psychology 71:390-405.

Evans-Pritchard, E.E. 1976. Witchcraft, oracles, and magic among the Azande (with an Introduction by Eva Gillies). Oxford: Clarendon.

Evans-Pritchard, E.E. 1981. Theorien über primitive Religionen. Frankfurt/M.: Suhrkamp.

Fischer, H. 1990. Völkerkunde im Nationalsozialismus. Aspekte der Anpassung, Affinität und Behauptung einer wissenschaftlichen Disziplin. Berlin: Reimer.

Hallpike, C.R. 1979. Foundations of primitive thoughts. Oxford: Oxford University Press.

Heinz, A. 1997. Savage thought and thoughtful savages. On the context of the evaluation of logical thought by Lévy-Bruhl and Evans-Pritchard. Anthropos 92:165-173.

Lévy-Bruhl, L. 1921. Das Denken der Naturvölker. Wien \& Leipzig: Braumüller.

Lévy-Bruhl, L. 1927. Die Geistige Welt der Primitiven. München: Bruckmann.

Pongratz, L.J. 1984. Problemgeschichte der Psychologie, 2. Aufl., München: Francke.

Ratnapalan, L. 2008. E. B. Tylor and the problem of primitive culture. History and Anthropology 19:131-142.

Razmyar, S., und C.L. Reeve. 2013. Individual differences in religiosity as a function of cognitive ability and cognitive style. Intelligence 41:667-673.

Schmidt, B.E. 2008. Einführung in die Religionsethnologie. Ideen und Konzepte. Berlin: Reimer.

Spencer, H. 1886. Die Principien der Psychologie. Bd. II. Stuttgart: Schweizerbart.

Spencer, H. 1887. Die Principien der Sociologie. Bd. II. Stuttgart: Schweizerbart.

Streck, B. 2013. Sterbendes Heidentum. Die Rekonstruktion der ersten Weltreligion. Leipzig: Eudora.

Vierkandt, A. 1937. Die entwicklungspsychologische Theorie der Zauberei. Archiv für die Gesamte Psychologie 98:420-489.

Werner, H. 1933. Einführung in die Entwicklungspsychologie, 2. Aufl., Leipzig: Barth.

Wolfradt, U. 2011. Ethnologie und Psychologie. Die Leipziger Schule der Völkerpsychologie. Berlin: Reimer.

Wolfradt, U. 2013. Der Einfluss des Entwicklungsgedankens nach Herbert Spencer auf Psychologie und Soziologie. In Die Entwicklung der Psyche in der Geschichte der Menschheit, Hrsg. G. Jüttemann, 81-92. Lengerich: Pabst.

Wolfradt, U., V. Oubaid, E.R. Straube, N. Bischoff, und J. Mischo. 1999. Thinking styles, schizotypal traits and anomalous experiences. Personality and Individual Differences 27:821-830. 
Wundt, W. 1905. Mythus und Religion. Teil 1. Völkerpsychologie. Eine Untersuchung von Sprache, Mythus und Sitte, Bd. 2. Leipzig: Barth.

Wundt, W. 1906. Mythus und Religion. Teil 2. Völkerpsychologie. Eine Untersuchung von Sprache, Mythus und Sitte, Bd. 2. Leipzig: Barth.

Wundt, W. 1913. Elemente der Völkerpsychologie. Grundlinien einer psychologischen Entwicklungsgeschichte der Menschheit, 2. Aufl., Leipzig: Barth. 